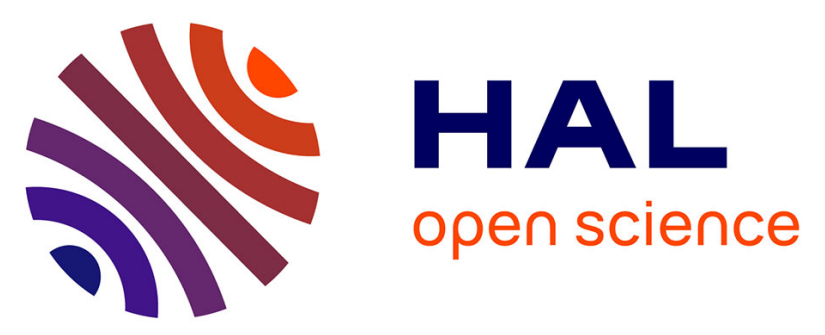

\title{
Fiber-draw-induced elongation and break-up of particles inside the core of a silica-based optical fiber
}

Manuel Vermillac, Jean-François Lupi, Francois Peters, Martiane Cabie, Philippe Vennegues, Courtney Kucera, T Neisius, J Ballato, Wilfried Blanc

\section{- To cite this version:}

Manuel Vermillac, Jean-François Lupi, Francois Peters, Martiane Cabie, Philippe Vennegues, et al.. Fiber-draw-induced elongation and break-up of particles inside the core of a silica-based optical fiber. Journal of the American Ceramic Society, 2017, 100 (5), pp.1814-1819. 10.1111/jace.14774 . hal01522905

\section{HAL Id: hal-01522905 \\ https://hal.science/hal-01522905}

Submitted on 14 Nov 2017

HAL is a multi-disciplinary open access archive for the deposit and dissemination of scientific research documents, whether they are published or not. The documents may come from teaching and research institutions in France or abroad, or from public or private research centers.
L'archive ouverte pluridisciplinaire HAL, est destinée au dépôt et à la diffusion de documents scientifiques de niveau recherche, publiés ou non, émanant des établissements d'enseignement et de recherche français ou étrangers, des laboratoires publics ou privés. 


\title{
Fiber-draw-induced elongation and break-up of particles inside the core of a silica-based optical fiber
}

\author{
M. Vermillac ${ }^{1}$ | J.-F. Lupi ${ }^{1}$ | F. Peters ${ }^{1}$ | M. Cabié ${ }^{2}$ | P. Vennéguès ${ }^{3}$ | C. Kucera ${ }^{4}$ | \\ T. Neisius ${ }^{2}$ | J. Ballato ${ }^{4}$ | W. Blanc ${ }^{1}$
}

${ }^{1}$ Université Côte d'Azur, CNRS, Institut de Physique de Nice site Valrose, UMR 7010, Parc Valrose, 06108, Nice,

Cedex 2, France

${ }^{2}$ Fédération des sciences chimiques de Marseille, Aix-Marseille Université CP2M CP2M, Marseille cedex 20, France

${ }^{3}$ Centre de Recherche sur l'Hétéro-

Hépitaxie et ses Applications, UPR CNRS 10, Valbonne Sophia Antipolis,

France

${ }^{4}$ Center for Optical Materials Science and Engineering Technologies (COMSET) and the Department of Materials Science and Engineering, Clemson University, Clemson, South Carolina 29634.

\section{Correspondence}

W. Blanc, Université Côte d'Azur, CNRS, Institut de Physique de Nice site Valrose, UMR 7010, Parc Valrose 06108 Nice,

Cedex 2, France.

Email: wilfried.blanc@unice.fr

Funding information

Agence Nationale de la Recherche, project Nice-DREAM, Grant/Award Number: ANR-14-CE07-0016-03.

\begin{abstract}
Particles in the core of optical fibers are widely studied to tailor or to improve optical properties. The analysis of nanoparticles embedded in silica-based optical fiber allowed new observations of the evolution of amorphous particles during fiber drawing. Even at the nanoscale, competition between viscous stresses and surface tension on the particles induces elongation and even break-up of particles during the process. Indeed, particles between 140 and $200 \mathrm{~nm}$ diameter inside the preform can break up in fragments with diameters down to $60 \mathrm{~nm}$ inside the drawn fiber. Break-up of particles appears as a new "top-down" strategy to produce small particles. These observations are promising for micro/nanostructured and multiphasic optical fibers.
\end{abstract}

\section{K E Y W O R D S}

nanoparticles, optical materials/properties, rheology/rheometry, silica

\section{1 | INTRODUCTION}

Nanoparticle-embedded glasses are particularly promising materials as they could overcome some limitations inherent to bulk materials or improve the versatility of single-phase materials. ${ }^{1}$ A paradigmatic example is the development of a new family of optical fiber lasers and amplifiers using luminescent ion-doped silica as a host glass. Nanoparticles in optical fibers can provide augmented intrinsic properties as they can combine the sturdiness and low cost of silica with particular spectroscopic behavior that would not appear in a pure silica local environment. ${ }^{2}$ As nanoparticles fully encapsulate the luminescent ions (such as rare-earth ions), they produce engineered spectroscopic properties. ${ }^{3,4}$ However, to avoid optical losses induced by light scattering, it is mandatory to control the size of particles. Another interest in nanoparticle-embedded optical fibers rely on their ability to develop random fiber lasers. ${ }^{5}$ Once again, optical properties of such lasers depend strongly on the size of the nanoparticles.

Heat-treatment of the fiber has been studied to control the nucleation/growth process, then to precisely tune the size of the resultant nanoparticles. ${ }^{6}$ However, this route tends to lessen the mechanical properties of the optical fiber. Accordingly, nanoparticles should already be present in the preform. However, thermodynamical and rheological effects are also known to modify the characteristics of the 
nanoparticles. Indeed, it has been reported recently that a thin rod of glass embedded in a polymer cladding can lead to the formation of nanoparticles. ${ }^{7,8}$ Such a structure is tailored through the formation of capillary instabilities induced by heat treatment. Cold-drawing of this structure can also be applied to form nanoparticles with controlled sizes. ${ }^{9}$ In this letter, we report on the evolution of nanoparticles during the fiber draw process and we show that fiber drawing itself can induce break-up of particles through rheological mechanisms. We demonstrate that it is possible to take advantage of this high-temperature elongation stage to control the size of the nanoparticles.

\section{2 | EXPERIMENTAL PROCEDURE}

A set of $10 \mathrm{~nm}-\mathrm{LaF}_{3}$ nanoparticles containing 0.1 at.\% $\mathrm{Tm}^{3+}$ was synthesized as is described in greater details elsewhere. ${ }^{3} 156 \mathrm{mg}$ of nanoparticles were dispersed in $100 \mathrm{~mL}$ of ethanol to elaborate the doping solution. The preform was fabricated by conventional MCVD (Modified Chemical Vapor Deposition) process. ${ }^{10} \mathrm{~A}$ Ge-doped silica porous layer of the core was immersed in $5 \mathrm{~mL}$ of the doping solution injected in the horizontally rotating tube ${ }^{11}$ and then dried at $1000^{\circ} \mathrm{C}$ under an oxygen gas flow. The porous layer was densified at $1800^{\circ} \mathrm{C}$. The tube was collapsed into the preform by heating above $2200^{\circ} \mathrm{C}$. The diameter of the preform was around $10 \mathrm{~mm}$ with a $1 \mathrm{~mm}$ core diameter. The optical fiber was drawn on a drawing tower by heating the preform at approximately $2000^{\circ} \mathrm{C}$ with a tension varying between 0.42 and $0.44 \mathrm{~N}$. The external diameter of the fiber was $125 \mu \mathrm{m}$. The compositions of the optical preform and fiber were measured using Energy Dispersive X-ray (EDX) and Wavelength Dispersive X-ray analyses. The average lanthanum and germanium concentration varies along the length (axial direction) of the preform between $0.4-2$ and $0.8-0.4$ at.\%, respectively. As observed previously, X-ray photoelectron spectroscopy measurements on preform confirm the evaporation of fluorine. ${ }^{12}$ The exact composition of the nanoparticles is still unknown but EDX measurements confirm that it is a Larich silicate phase as $\mathrm{La}$ content was measured to be 18 at. \% in a micrometric particle.

To investigate the shape of the particles in the drawing direction, the 3D technique of focused ion beam (FIB) tomography was used. ${ }^{13}$ 3D analysis was conducted on a dual beam Helios 600 NanoLab manufactured by FEI (Hillsboro, Oregon, USA). The principle is to alternatively remove slices of material by FIB milling and take a 2D scanning electron microscopy (SEM) image of each section. The acquired stack of 2D images is then processed to reconstruct the volume. Two volumes of the core of the fiber were reconstructed with dimensions of $5 \mu \mathrm{m} \times 5 \mu \mathrm{m} \times 0.5 \mu \mathrm{m}$ and
$10 \mu \mathrm{m} \times 5 \mu \mathrm{m} \times 0.5 \mu \mathrm{m}$, respectively. The distance between two slices was $5 \mathrm{~nm}$.

\section{3 | RESULTS}

Longitudinal sections of the preform and the fiber were analyzed. The SEM pictures are reported in Figure 1. From the preform to the fiber, observed are modifications of the shape of the particles. They are mainly circular in the preform while noncircular shapes are clearly visible in the fiber. In this 2D image, elongated particles with sharp ends are observed. This shape is due to the angle $\left(4^{\circ}\right)$ between the drawing axis of the fiber and the probed SEM surface.

To better understand these particles in the fiber, a 3D reconstruction is presented in Figure 2. It is the volume reconstruction of the core of the optical fiber based on the FIB and SEM analysis. The host matrix was subtracted from the 3D rendering to display the lanthanum-rich amorphous particles. It should be noted that the present reconstruction is obtained by plotting particles with a radius bigger than $10 \mathrm{~nm}$. The volume fraction of particles from tomography is estimated to be around 5\%. Several patterns (labeled by numbers identified in Figure 2) can be identified in this tomograph: long cylinders (some terminated with pointy end as already observed in the 2D picture) (i), chains of particles, with small particles homogeneously spaced (ii), particles with diameter variation along their lengths (iii), coalescence of nanoparticles (iv), isolated spherical particles (v).

\section{4 | DISCUSSIONS}

All these patterns are also present in the second SEM/FIB analyzed volume and are attributed to the drawing step. During this stage, the silica-based preform is heated up to $2000^{\circ} \mathrm{C}$. As it is above its softening temperature, the glass flows. Since the deformation is mainly extensional, ${ }^{14}$ the force applied on the fiber during the drawing is,

$$
F=3 \eta(z) \pi R_{\mathrm{f}}^{2}(z) \frac{\partial V_{z}}{\partial z}
$$

with $z$ referring to the position of the fiber inside the area of deformation, $R_{\mathrm{f}}(z)$ is the radius of the preform/fiber at the $\mathrm{z}$ position, $\eta$ is the viscosity and $V_{z}$ the velocity in the flow direction. The deformation rate $\dot{\epsilon}$ is defined as,

$$
\dot{\boldsymbol{\epsilon}}=\frac{\partial V_{z}}{\partial \mathrm{z}}=\frac{-2 Q R_{f}^{\prime}(z)}{R_{f}^{3}(z)}
$$

where $R_{\mathrm{f}}^{\prime}(z)$ is the derivative of the radius with respect to $z$ and the flow rate $Q$, with, 


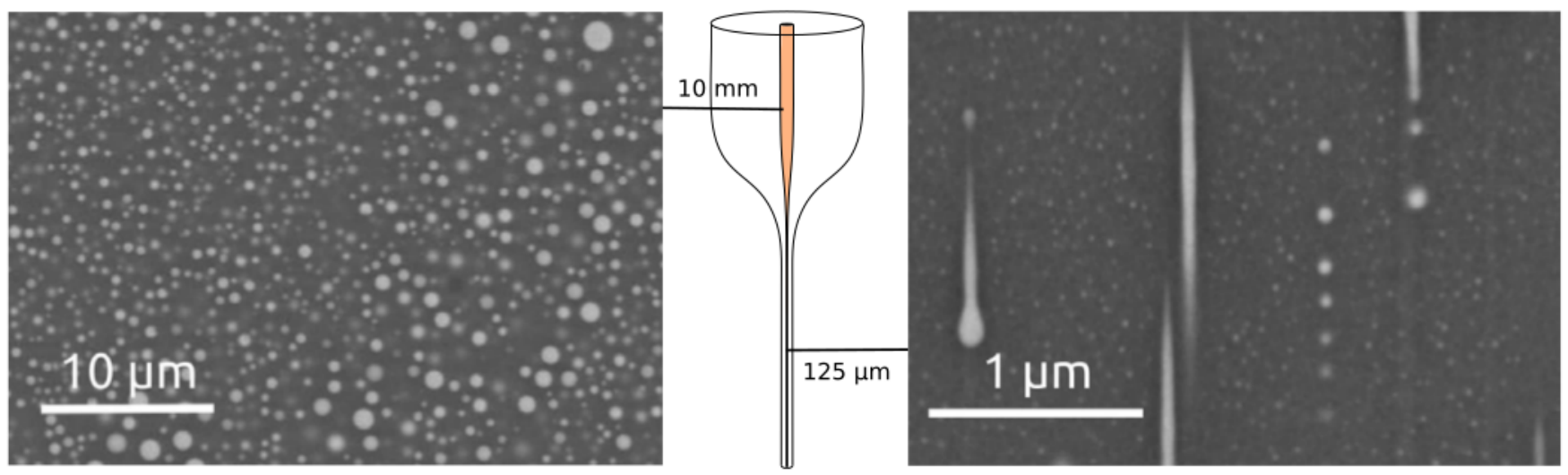

F IG URE 1 SEM pictures of longitudinal sections corresponding to the preform (left) and the fiber (right) [Color figure can be viewed at wileyonlinelibrary.com]

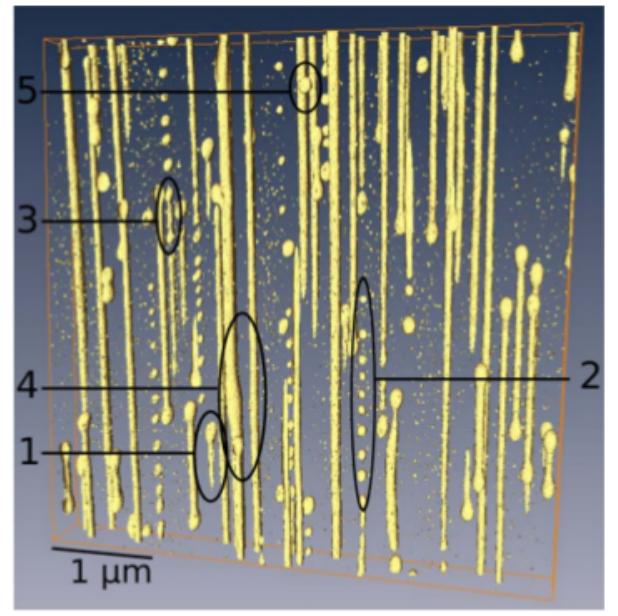

FIGURE 2 3D reconstruction of the volume of the core of the optical fiber analyzed by FIB and SEM. The host matrix (mainly silica) and particles with radius less than $10 \mathrm{~nm}$ were removed. For the numbers, see the text. The drawing axis is vertical [Color figure can be viewed at wileyonlinelibrary.com]

$$
Q=Q(z)=V(z) S(z)
$$

where $S(z)$ is the section of the fiber at the position $z$. Then, as the radius evolves during the drawing (from $10 \mathrm{~mm}$ to $125 \mu \mathrm{m}$ ), the deformation rate $\dot{\epsilon}$ changes also. As a consequence, all along the drawing, the viscous stress $\sigma$ evolves as,

$$
\sigma=\frac{F}{\pi R_{f}^{2}(z)}=3 \eta \dot{\epsilon}
$$

where $\eta$ is the viscosity (here, it is the viscosity of the core of the optical fiber). Then, the shape of the amorphous particles can be interpreted in the frame of elongation due to a flow-induced deformation. ${ }^{15}$ Indeed, it is neither relaxation nor solid deformation but the viscous flow of the material during the fiber drawing that induces this structure. ${ }^{15}$ More precisely, this structure comes from the competition between viscous stress of the host matrix that tends to elongate particles and surface tension of the particles that tends to minimize their surface during the flow of the glass (then to maintain a spherical shape). These rheological considerations were demonstrated for microparticles but remain valid even at a scale of tens of nanometers. ${ }^{16}$

Effects of the flow on the nanoparticles can be expressed depending on the capillary number $\mathrm{Ca}$ which is size-dependent,

$$
\mathrm{Ca}=\frac{\sigma R_{\mathrm{NP}}}{\gamma}
$$

where $R_{\mathrm{NP}}$ is the radius of the particle and $\gamma$ the surface tension between the particle and the matrix. For a determined flow, the higher the capillary number (or larger the particles), the more they can deform. This deformation is commonly quantified by the Taylor parameter,

$$
D=\frac{L-B}{L+B}
$$

where $L$ and $B$ are the large and small axis of the ellipsoidal-like elongated particle, respectively.

Figure 3 shows the deformation of particles inside the fiber as a function of their volume-equivalent-sphere radius (assuming that all the particles are initially spherical and can only deform during the drawing process; i.e., nanoparticles cannot dissolve or grow). This size parameter is proportional to the capillary number as one neglects viscosity and surface tension size dependence. We emphasize that these reported deformations are not equilibrium shapes since $\mathrm{Ca}$ increases through the process and the glass is quenched. Only particles fully included in the volume represented in Figure 2 are plotted in Figure 3. The particles are sorted in different groups according to their shapes: separated particles in chains produced by break-up (blue squares), isolated particles (yellow circles), highly 
FIGURE 3 Taylor parameter D as a function of the volume-equivalent-sphere radius of particles. Blue squares: separated particles in chains produced by break-up (2), yellow circles: isolated particles (5), red diamonds: highly elongated particles (1) and green triangles: elongated particles with pronounced Rayleigh instabilities (3) (numbers in parentheses are related given in Figure 2) [Color figure can be viewed at wileyonlinelibrary.com]

FIGURE 4 Size distribution of radius of particles produced by break-up. Sample 1 and 2 correspond to both volumes analyzed [Color figure can be viewed at wileyonlinelibrary.com]
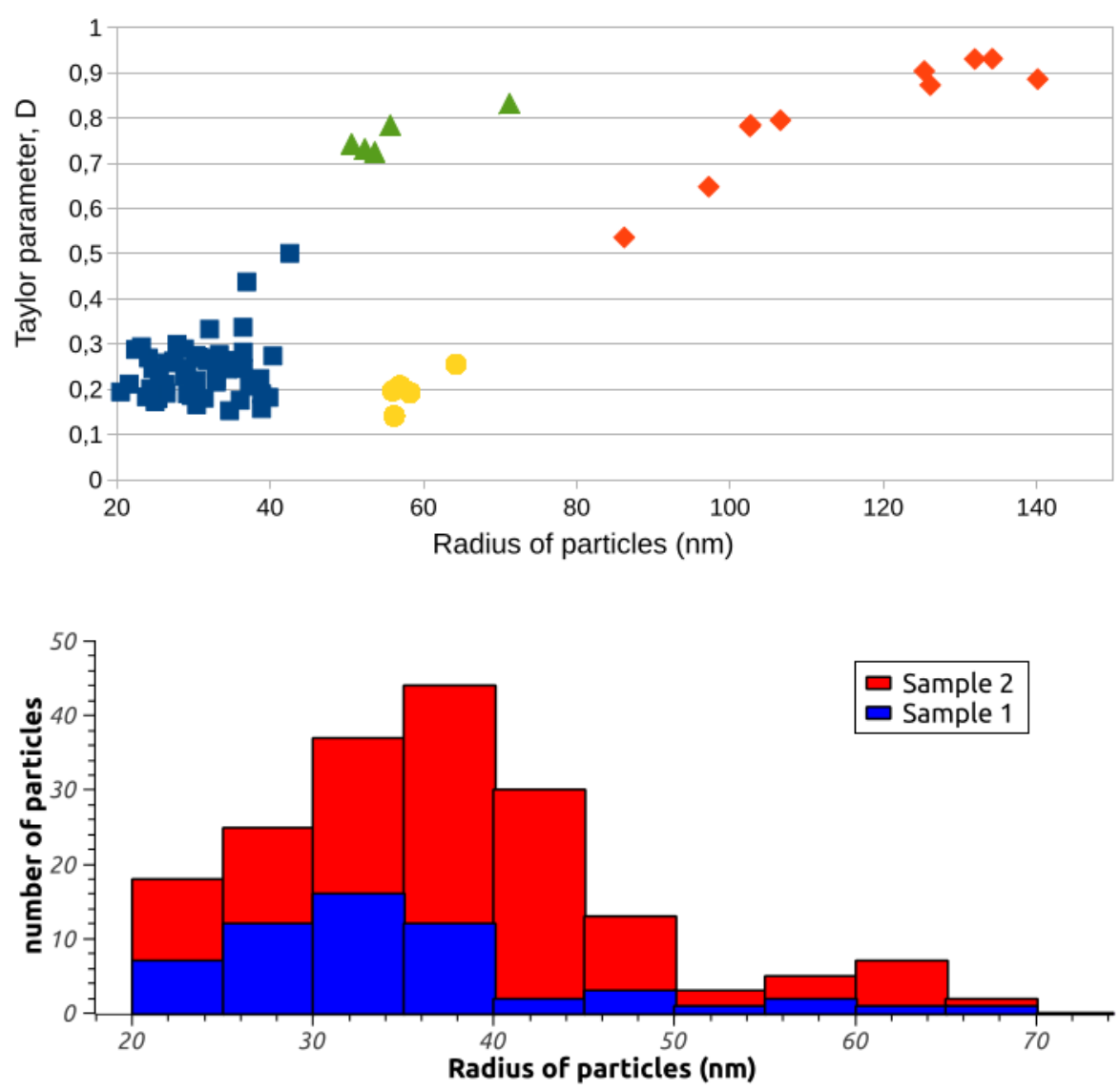

elongated particles (red diamonds), and elongated particles with pronounced Rayleigh instabilities (green triangles).

Long cylinders in Figure 3 (red diamonds) are spherical particles from the preform that deform under the viscous stresses of the fiber drawing. The highest deformation ratio (D) measured is about 0.9. However, a higher deformation ratio is expected but it cannot be measured with these analyses as the longer cylinders are not fully included in the volume probed. Considering their high elongation, breakup is not achieved because of the quenching of the glass and ceasing of deformation.

Particles that elongate enough undergo Rayleigh instabilities during the fiber drawing that induce the break-up of particles. ${ }^{15}$ Break-up produces chains of homogeneously spaced particles with monodisperse diameters (blue squares in Figure 3). The size of the initial particles in the preform that induces full break-up can be estimated by summing the volume of all particles forming each chains. From smallest and largest chains of particles measured in the fiber, the break-up occurs for particles in the preform whose radius is between 70 and $110 \mathrm{~nm}$. Figure 4 provides the size distribution of radius of particles that are produced through break-up and analyzed on both volumes. Size of particles is centered around $30-40 \mathrm{~nm}$ of radius. It is interesting to note that it has been reported that $50 \mathrm{~nm}$ nanoparticles are compatible with a threshold for transparency. ${ }^{17}$
For a few cases, all the particles in the chains are not completely separated (eg, case 3 in Figure 2). Indeed, some particles comprised in the chains have a high degree of deformation (green triangles in Figure 3) because the flow ceased while they were on the verge of breaking-up due to Rayleigh instability. Except for these transient particles, those smaller than $120 \mathrm{~nm}$ (yellow and blue data in Figure 3) are almost spherical because surface tension effects are stronger for small particles.

To induce break-up of particles, two conditions are necessary: first, the capillary number must be high enough to overcome surface tension, second, a sufficient deformation is necessary to initiate the Rayleigh instabilities. As the drawing stage is not stationary but dynamic (temperature quenching and change in the radius), for small particles (radius less than $70 \mathrm{~nm}$ ), there is a longer delay for viscous stresses (i.e., Ca) to become sufficient and thus less remaining deformation to break-up. For large particles (radius $>110 \mathrm{~nm}$ ), break-up dynamics initiate earlier, but the larger they are, the longer it takes. This is the reason that only a certain range of particles can break-up.

Break-up of particles is a complicated process that depends on the elongation history and on the value of the matrix and particles viscosities. In the present case, due to temperature variations through the process (from $2000^{\circ} \mathrm{C}$ to room temperature), the viscosity, the deformation rate, and 
likely surface tension change as the material is drawn. In addition the viscous stress $\sigma$ continuously increases during fiber drawing. For those reasons a precise analysis is not easy. However, experimental studies of drop break-up in homogeneous and stationary extensional flow have shown that radius of particles produced by break-up decreases by increasing $\mathrm{Ca}$ or increasing the ratio of viscosity between the particle and the matrix. ${ }^{18}$ Thus, for a defined material, increasing the fiber drawing tension may be a good way to produce smaller droplets. By decreasing the drawing temperature, it is possible to increase the tension. For a temperature of $1900^{\circ} \mathrm{C}$, the draw tension is reported to be about $7 \mathrm{~N},{ }^{19}$ that is about 16 times more than the tension used to prepare our fibers. This increase is also a possibility to lower the diameter of fragments and improve the kinetics.

Except for the ability to control the size, changing the shape of nanoparticles is also of interest. From previous experiments, it is possible to elongate a particle up to about 200 times its initial diameter before break-up. ${ }^{15}$ Even less deformation can lead to important changes in optical properties in the case of semiconductor ${ }^{20}$ and metallic 21 nanoparticles of interest in optical fibers. ${ }^{22}$ Indeed, energy bandgap (semiconductor nanoparticles) and surface plasmon resonances (metallic nanoparticles) are strongly affected by the size and shape of the particles.

Regarding light scattering, the scattering cross section of nanoparticles increases as $R^{6}$ in the frame of the Rayleigh scattering approach (nanoparticles ten times smaller than the wavelength of light). Then, the break-up mechanism is of great interest as it reduces the radius of the particles which lessens optical losses. Moreover, when the volume fraction of nanoparticles increases, the "independent scattering regime" approximation is no longer valid and nanoparticles will induce light interferences. Once again, aligned particles, as those produced by break-up, were reported to be beneficial. ${ }^{23}$ Finally, optical losses are affected by the elongated profile of the particles. ${ }^{24}$ The effect is minor considering the high optical losses which could exceed $100 \mathrm{~dB} / \mathrm{m}$ and size of particles in these fibers.

\section{5 | CONCLUSION}

In conclusion, we have fabricated a silica-based optical preform containing lanthanum-rich particles inside its core. The optical fiber was then obtained by heating and pulling the preform. During the flow of the material, viscous stresses induced deformation of the particles into long threads. Another observation is that a part of the particles undergone break-up. These phenomena clearly offer new possibilities for the control of the size and shape of particles with beneficial interest for optical property tailoring. This work also highlights that scanning electron microscopy measurements on transverse sections of the optical fibers, as usually reported in the literature, are not sufficient to characterize the shape and size distribution of particles.

\section{ACKNOWLEDGMENTS}

We acknowledge S. Trzesien and M. Ude (LPMC, Nice) for the fabrication of the samples and E. Lemaire, C. Raufaste, and Y. Bouret (LPMC, Nice) for their helpful discussions. This work was supported by ANR project Nice-DREAM (ANR-14-CE07-0016-03).

\section{REFERENCES}

1. Gonçalves MC, Santos LF, Almeida RM. Rare-earth-doped transparent glass ceramics. C R Chim. 2002;5:845-854.

2. Blanc W, Dussardier B. Formation and applications of nanoparticles in silica optical fibers. J Opt. 2016;45:247-254.

3. Kucera C, Kokuoz B, Edmondson D, et al. Designer emission spectra through tailored energy transfer in nanoparticle-doped silica preforms. Opt Lett. 2009;34:2339-2341.

4. d'Acapito F, Blanc W, Dussardier B. Different $\mathrm{Er}^{3+}$ environments in Mg-based nanoparticle-doped optical fibre preforms. J NonCryst Solids. 2014;401:50-53.

5. Cao H. Lasing in random media. Waves Random Media. 2003;13:R1-R39.

6. Tick P, Borrelli N, Reaney I. The relationship between structure and transparency in glass-ceramic materials. Opt Mater. 2000;15:81-91.

7. Kaufman JJ, Tao G, Shabahang S, et al. Structured spheres generated by an in-fibre fluid instability. Nature. 2012;487:463-467.

8. Shabahang S, Kaufman J, Deng D, Abouraddy A. Observation of the plateau-rayleigh capillary instability in multimaterial optical fibers. Appl Phys Lett. 2011;99:161909.

9. Shabahang S, Tao G, Kaufman JJ, et al. Controlled fragmentation of multimaterial fibres and films via polymer cold-drawing. Nature. 2016;534:529-533.

10. MacChesney $\mathrm{J}, \mathrm{O}^{\prime}$ connor P, Presby H. A new technique for the preparation of low-loss and graded-index optical fibers. Proc IEEE. 1974;62:1280-1281.

11. Sysala O, Kasik I, Spejtkova I. Preparation of preforms and optical fibres containing aluminium by the solution-doping method. Ceramics. 1991;35:363-367.

12. Diamente PR, Raudsepp M, van Veggel FC. Dispersible $\mathrm{Tm}^{3+}$ doped nanoparticles that exhibit strong $1.47 \mu \mathrm{m}$ photoluminescence. Adv Funct Mater. 2007;17:363-368.

13. Holzer L, Indutnyi F, Gasser P, Münch B, Wegmann M. Threedimensional analysis of porous BaTio3 ceramics using fib nanotomography. J Microsc. 2004;216:84-95.

14. Paek U, Runk R. Physical behavior of the neck-down region during furnace drawing of silica fibers. J Appl Phys. 1978;49:44174422.

15. Grace HP. Dispersion phenomena in high viscosity immiscible fluid systems and application of static mixers as dispersion devices in such systems. Chem Eng Commun. 1982;14:225277. 
16. Squires TM, Quake SR. Microfluidics: fluid physics at the nanoliter scale. Rev Mod Phys. 2005;77:977.

17. Blanc W, Mauroy V, Nguyen L, et al. Fabrication of rare earth-doped transparent glass ceramic optical fibers by modified chemical vapor deposition. J Am Ceram Soc. 2011;94:23152318.

18. Mikami T, Cox R, Mason S. Breakup of extending liquid threads. Int J Multiphase Flow. 1975;2:113-138.

19. Paek U-C, Schroeder C, Kurkjian CR. Determination of the viscosity of high silica glasses during fibre drawing. Glass Technol. 1988;29:263-266.

20. Peng X, Manna L, Yang W, et al. Shape control of CdSe nanocrystals. Nature. 2000;404:59-61.

21. Kelly KL, Coronado E, Zhao LL, Schatz GC. The optical properties of metal nanoparticles: the influence of size, shape, and dielectric environment. J Phys Chem B. 2003;107:668-677.
22. Ju S, Nguyen VL, Watekar PR, et al. Fabrication and optical characteristics of a novel optical fiber doped with the $\mathrm{Au}$ nanoparticles. J Nanosci Nanotechnol. 2006;6:3555-3558.

23. Ivezić Z, Mengüç MP. An investigation of dependent/independent scattering regimes using a discrete dipole approximation. Int $J$ Heat Mass Transfer. 1996;39:811-822.

24. Van De Hulst H, Light scattering by small particles. New York, NY: Courier Corporation; 1957. 